PHYSICAL REVIEW D 94, 049907(E) (2016)

\title{
Publisher's Note: What gravity waves are telling about quantum spacetime [Phys. Rev. D 93, 124065 (2016)]
}

\author{
Michele Arzano and Gianluca Calcagni \\ (Received 15 August 2016; published 26 August 2016)
}

DOI: 10.1103/PhysRevD.94.049907

This paper was published online on 27 June 2016 with an incorrect sentence in the Acknowledgments. The last sentence of the Acknowledgments should read as "The work of G.C. is under a Ramón y Cajal contract and is supported by the I + D grant FIS2014-54800-C2-2-P.” The Acknowledgments have been corrected as of 19 August 2016. The Acknowledgments are incorrect in the printed version of the journal. 